\title{
IIoT Framework Based ML Model to Improve Automobile Industry Product
}

\author{
S. Gopalakrishnan ${ }^{1, *}$ and M. Senthil Kumaran ${ }^{2}$ \\ ${ }^{1}$ Research Scholar, Anna University, Chennai, Tamilnadu, India \\ ${ }^{2}$ Department of EEE, SSN College of Engineering, Chennai, Tamilnadu, India \\ *Corresponding Author: S. Gopalakrishnan Email: gopal.pgsk@gmail.com \\ Received: 02 June 2021; Accepted: 11 July 2021
}

\begin{abstract}
In the automotive industry, multiple predictive maintenance units run behind the scenes in every production process to support significant product development, particularly among Accessories Manufacturers (AMs). As a result, they wish to maintain a positive relationship with vehicle manufacturers by providing 100 percent quality assurances for accessories. This is only achievable if they implement an effective anticipatory strategy that prioritizes quality control before and after product development. To do this, many sensors devices are interconnected in the production area to collect operational data (humanity, viscosity, and force) continuously received from machines and sent to backend computers for control operations and predictive analysis. As a result, there is a vast volume of data that may be processed further to obtain accurate information on equipment processing speed and production efficiency. However, extracting details in the essential format for data-driven decision support for predictive maintenance is problematic. As a result, an effective predictive maintenance approach based on Machine Learning (ML) methods is established. It has an impact on the Hybrid Machine Learning (HML) model, which blends supervised and unsupervised learning. It helps to forecast breakdowns and production line deviations ahead of time, preventing the manufacturing unit from shutting down. The proposed predictive methodology has been tested in terms of earlier anomaly detection, production line accuracy \& machinery efficiency and compared with other existing ML based predictive maintenance approaches.
\end{abstract}

Keywords: Low power sensors; predictive maintenance; industrial ioT; hybrid ML model

\section{Introduction}

Every year, an automobile industry increases the production of the commercial vehicles (trucks, trailers and buses) and passenger vehicles (cars and other four wheelers) [1,2]. For example, automobile statistical census announced that nearly 90 million vehicles have been launched at the end of 2020. So, day-by-day, the production value is growing rapidly however the manufactured vehicles has not reached $100 \%$ effectively because of untested low quality accessories procurement from the third party accessories manufacturing vendors $[3,4]$. In the production phase, they directly involved purchased accessories in the newly

This work is licensed under a Creative Commons Attribution 4.0 International License, which permits unrestricted use, distribution, and reproduction in any medium, provided the original work is properly cited. 
lunching vehicles. For every accessories component should maintained test phase data and it has easily track if it is saved in the serve through internet of things (IoT) platform $[5,6]$. But small companies they didn't follow IoT for storing the test data values which seriously opposing in the accessories standard. According to the KPMG's Global Automotive Executive Survey report (2018) says that out of 100 vehicles $2 \%$ of vehicles hold defective components which causes serious issues like earlier failure of vehicle parts and seriously affect the road safety of the country [7]. It seriously damaged the standard quality policy of the particular accessories manufacturing company. There are several reason behinds this, (i) faculty purchase from material vendors (ii) purchase engineer directly send the materials to accessories production phase (iii) lack of acquires of the employees. Therefore, a recent automotive industry has maintained active and protective the manufacturing standard between material vendors and production employees. It is achievable only if the data processing is successful integrated from the factory floor which plays a key role in optimizing the manufacturing and business processes as well as generating business values for the manufacturers. Furthermore, to enhance the contemporary demands of the market with short product lifetime, the industrial systems and processes need to be monitored and supervised continuously often to meet customer's requirements and market competition. Recently, Industrial Internet of things (IIOT) framework enters in all sectors of automobile industries for predictive maintenance by placing smart sensor devices to do sensitivity operation includes monitoring and tracking is primary tasks prominently [8-12]. It has flexible structure to incorporate multiple tasks and scale exponentially by interfacing different embedded devices. In Fig. 1 represents new components accompanying for cyber physical system (CPS) for predictive maintenance [13-15]. The sensed data is properly handled and extract the required field from raw data from instant machine. The machine learning approach is applied to extract optimum feature and ensure the production machine quality after evaluating required parameters which is shown digital display mounted on each individual machine at every station wing [16]. Hence, it conveys prior information about machine production quality, efficiency period and improved productivity [17,18]. The contribution of this paper is to develop an effective predictive maintenance system by influence with hybrid ML model in the IIoT framework. It induces smart sensorkit for continuous collection of machine data instantly and ensures secured content saving at the centre cloud for further reference.

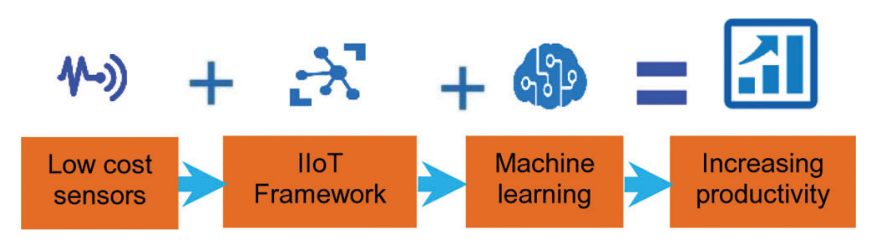

Figure 1: Some functions of ${ }^{x}$

The proposed IIoT framework have silent features includes (i) IPv6 over low-power sensor kit interface gives synchronized sensed data-acquisition, (ii) the hybrid ML model leads earlier detection of process deviation as well as anomaly detection within stipulated time and (iii) an optimum classifier is generated for each sensor by learning its historical data. The paper is organized as follows: Section 1 explains current scenario of automobile industries and mentioned important of predictive maintenance especially Accessories Manufacturers (AMs) sectors. Section 2 describes the proposed IIoT framework for predictive maintenance pilot. Then, the hybrid ML model is discussed in detail and its data flow and processing operation of each individual phase is described in Section 3. And Section 4 shows the experimental result of proposed system which is compared with existing strategies. Finally, the conclusion has been made from the comparison result in Section 5. 


\section{Related Works}

Vijender Solanki et al. [19] proposed a new IoT framework for vehicle monitoring and technical status of vehicle parts which provides accurate scheduling period for vehicle service. Thus, it improves proper functioning capacity of the vehicle which in turn enhanced the lifetime as well as resale market value. It also supported with automatic vehicle traffic monitoring system, where it can direct the vehicle towards shortest path. Thereby, the vehicle reaches centre faster and get quick service thus, minimized the delay delivery to customer after vehicle completed its service. Each data of individual parts are recorded in the cloud storage, hence, both customer and authorized service centre head can viewed the current status of the vehicle which gives the transparency of service work and quality assurance. According to the proposed framework, created a new area for vehicle service using advance monitoring technology measures accurate detailing of vehicle lubricant and speed for good mileage etc. Based on that, vehicle called for service and fine turns the mileage capability for customer satisfaction. However, initialize cost of installation is too high and continues calibration of machine is needed to compute right parametric value. Chowdhury et al. [20] addressed inbuilt fault detection using mobile based application, it gives smart prediction of vehicle vibration and indicate passenger uncomfortable to vehicle owner. So, earlier corrective action may take and thus, lead safe and comfortable travel. This system is most useful for vehicle cab service and other travelling agents. The web application is utilized instead of placing separate sensor to read the data of about the vehicle. Because, whenever vehicle owner wants to know about the vehicle performance, then, it can easily track from stored data of previous service history which is ready available in the cloud. By using this web application, vehicle owner can be identified Level-1 failure and suggestion can give from data-driven approach to vehicle owner of vehicle manageable period. Zhen Chen et al. [21] designed SPC based predicted maintenance model for analyzing machine health condition. It is achieved by generated optimistic values within stipulated time which minimize the cost value per vehicle cycle under observation. It has wide analysis wing towards measuring vehicles health condition includes index, frequency, calibration strength and time. Based on these values, control chart is generated and reduces the chance of occurring machine failure and performance degradation. The proposed model has reduced unnecessary production shutdown and prior intimation about machine service. The service cycle is assigned automatically, suggestion of new vehicle accessories is allotted after superimposed X control chart on the individual vehicle. Thereby, it has increased the vehicle ageing and extended the market value. Herr et al. [22] discussed the optimization problem from large data volume accumulated in the Prognostics and Health Management (PHM) system.

It involves both the assignment of missions and initiate service scheduling only the machine crossed the threshold limit point. The detailed mathematical description is discussed to show how the objective function gives minimized optimum value. The continuous monitoring suffers lifetime of sensors where physical active but sensing function is degraded, thus, may given wrong dataset of the analyzing machine as well as observed vehicle. The optimized values access the machine condition in right way and properly accelerated the control signal at emergency time. However, computational complexity is extended thus, reduces quality performance of the checking machine. Nagi Z. Gebraeel et al. [23] updated the predictive maintenance policy after evaluation dataset received from embedded device. The degraded signal is activated and reportable only if the value is sensed below the expected threshold limit. The characteristic of individual component is evaluated and periodically updated in the real time monitoring system. That means, residual life distribution (RLD) is continuously updating with relevant data driven model. Since, it gives probability nature of occurring failures and improved the predictive maintenance policy. The assured quality service of vehicle is derived and continued still the next service is permitted. This model is quite simple and applicable to the small-scale manufacturing sector. The sensitive parameter ratio is carried out from sensors raw data, provide quality assurance to the vehicle vendors. Satta et al. [24] reviewed taxonomies and recent methodology involved for vehicle predictive maintenance. Several appliance dataset is 
analyzed to extract the actual figure out the failure root cause. It is simply comparing previous dataset with the instant dataset from observed machine and vehicle. It has projected dissimilarities among all appliances and identifies mutual behaviors degradation. It can easily detect the upcoming failure by concurrent mutual differences from the cohort. In this study, identified the ways of occurring new faulty features of the appliance signal. It sets global identical survey of anomaly behaviors appearance during vehicle under observation. It takes all possible feature dataset and stored on the cloud space for further analysis. If it is too high in volume then gives proper priority assignment procedure is followed to reduce the data count. Thus, bring easy accessibility of data whenever client and authorized person want to know about the machine and vehicle condition. Emir et al. [25] proposed smart car system based on IoT technology track the quality conditions of the vehicle at any time circumstance and calibrate appropriate feature dataset for earlier anomaly detection.

It contains a standard an-board-diagnostic (OBD) provides information about the vehicle inbuilt component function. Everything is connected with web application and accessible in IoT platform. It enables electronics monitoring system which receivers control signal after scanning tools effectively utilized the acquired vehicle data. The vehicle contained IBM BLUEMIX cloud storage where recent application data is saved. It is highly involved for measuring vehicle working operation and evaluates the component current status. Both smartphone and sensors jointly the feature extraction which has accelerated required control signal for improvement of the vehicle life period. The smartphone directly connected with embedded system of the vehicle through USB or Bluetooth. The IBM BLUEMIX cloud act as temporary storage for all extracted data feature. But it is limited in storage hence, regular updating is possible by renovating of past history database.

\section{Industrial IoT Frameworks}

Recently, a lot of innovation ideas brought for developing industrial 4.0 environment set up for predictive maintenance system in order to do continuous monitoring on machines performance accuracy and quality product outlet. It is possible by updating existing technology with recent advancement in the devices (smart sensors) which support high quality observer function that continuously collect the sensed data from the environment. Since, it has involved inter-connection of many digital devices which requires proper coordination must be established through Industrial Internet of Things (IIoT) platform integrated with wireless sensor network. It provides flexible connectivity among internal and external device where it leads to have quality predicative maintenance system. According to this, machine data can be easily accessible to each other and provide proper gateway between the machine supervisor and other terrestrial networks for data communication. The actual status of machine is monitored and fetches as internal data which can further process at programmable logic controller and get the required dataset feeder to the maintenance unit for earlier identification of root causes (process deviation, sensitivity, specificity) by previous fault feature database. A huge amount of sensed data extracted from machine and processed to know about the specific feature. Whenever, data volume is large, then, it acquires sufficient processing time and leads more memory space to store data. Thereby, two separate spaces are allocated for internal and external data as a local server and centre cloud. However, an efficient open interface introduced between sensor and embedded device.

Thus, ensure the proper examination carried on acquired data and forward to control centre for preprocessing data occurred. It creates two layers (Sensor-to-Fog and Fog-to-Fog) for data sharing which minimize number of data in cloud significantly. Because, in the first layer (Sensor-to-Fog), almost all possible data is processed and identify most essential data which is forward into second layer (Fog-toFog). Again, data processing is carried until if it is required. Then, it allows limited amount of data move in the cloud for further data analysis. The performance accuracy of the IIoT based network architecture is significantly minimizes energy constrains and maximize the prediction accuracy especially in the place of 
data monitoring mandatory. In Fig. 2 shows implemented IIoT-architecture for proposed predictive maintenance system. It consists of three layers namely device connectivity, data analytic and business connectivity. The proper gateway is established among sensorkit for receiving machine data which is stored in local server in that feature comparison is done with respect to previous database. If it is different feature, then, it will stored in the historical database located in the centre cloud (Azure) for further stream analysis carried to get clear characteristic about instant machine data. The machine learning algorithm finds optimist solution and computes dataset for upcoming data. Finally, if the training part is completed successfully, then, stream analysis is made in the web hot for a real time evaluation of the machine as well as product quality. Furthermore, anomaly detection notification is shared with maintenance system where necessary corrective measurement is carried over for maintaining standard performance accuracy. The following section gives an overview of the ML models that were used.

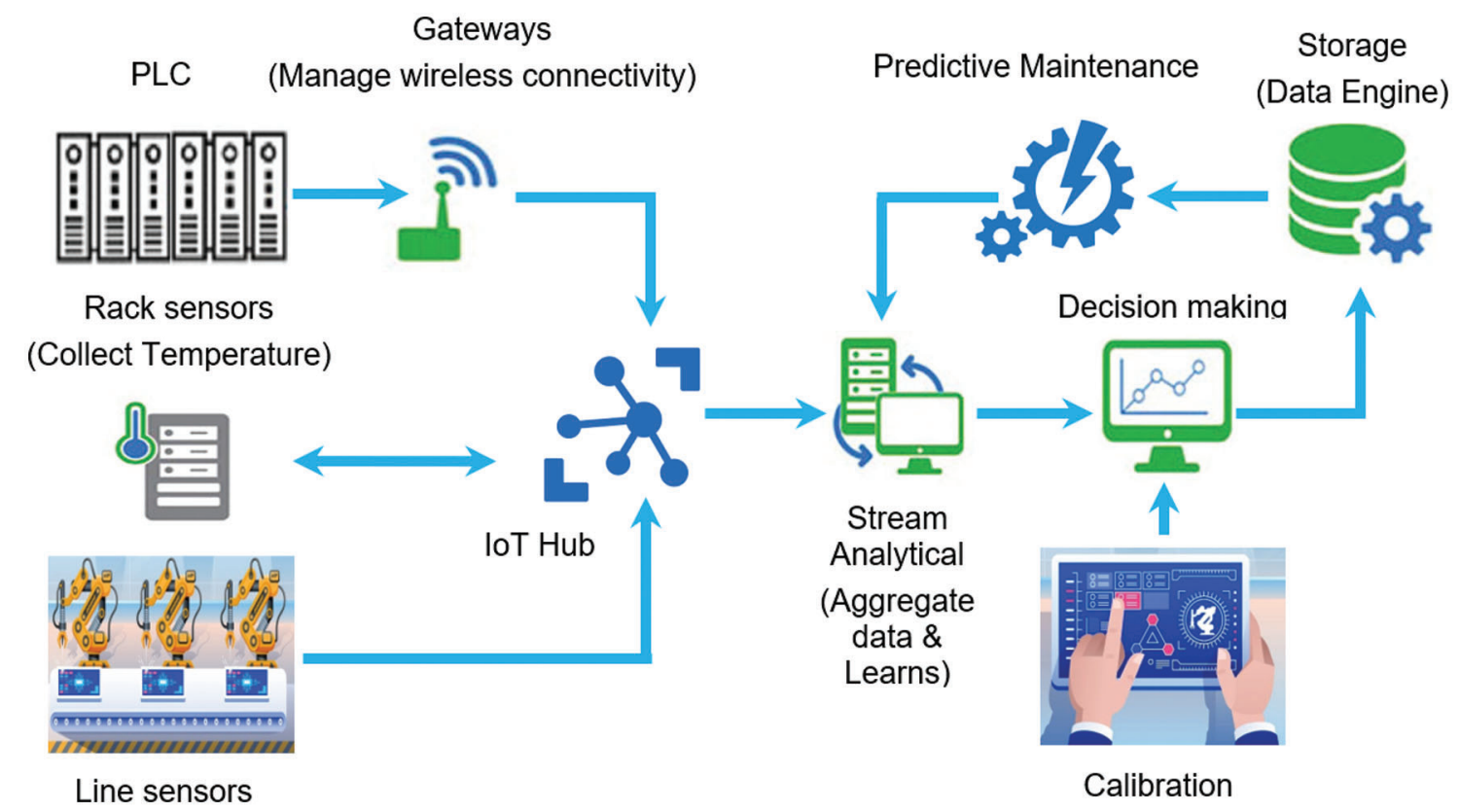

Figure 2: Implemented IIoT-architecture for predictive maintenance

\section{Improved Hybrid ML Strategy}

The hybrid machine learning strategy is carried out in the proposed IIoT framework for improving earlier detection of anomaly presence in the product as well as machine process deviation. In Fig. 3 gives a hybrid machine learning strategy for predictive maintenance pilot. The predictive maintenance system works under ML approach for segregating an optimistic dataset for control signal whenever finds earlier process deviation root causes occurs. Because, the proposed model consist of two major parts namely training and running phase where actual evaluation of about machine and product performed. In Fig. 4 convey data flow and processing operation of each individual phase is described. In the training phase, deals with the production efficiency of the manufacturing machine module at the operating time and ensures the quality outcome of the product before leave from the production line. It is obtained by evaluating three important parameters such as sensitivity, specificity and accuracy. The historical database is involved for comparing previous dataset with current dataset. if new data is identified for process deviation then it will be stored in the historical dataset in the cloud. In such case, un-supervised learning is trigged to get the data for future reference. Whenever, usual data feature receives from instant machine 
data is simply connected with hard class assignment which is internally joined the supervised learning model. Because, feature comparison is quite normal in case of supervised learning where individual product ID number is taken as labelled value. It is easily accessible by maintenance system after verifying identification code generated randomly at the time of product investigation. Sub sequentially, running phase concerned for the quantitative evaluation of the instant product data with historical database to acquire a new set of data which helps for earlier detection of the product failure. The feature extraction module is involved to track the shapes and weight of the product and its value is stored in the both local and cloud space for future customer claims about the product failure. The data analytics of both phases is briefly described in the following section along with mathematical description of each module.

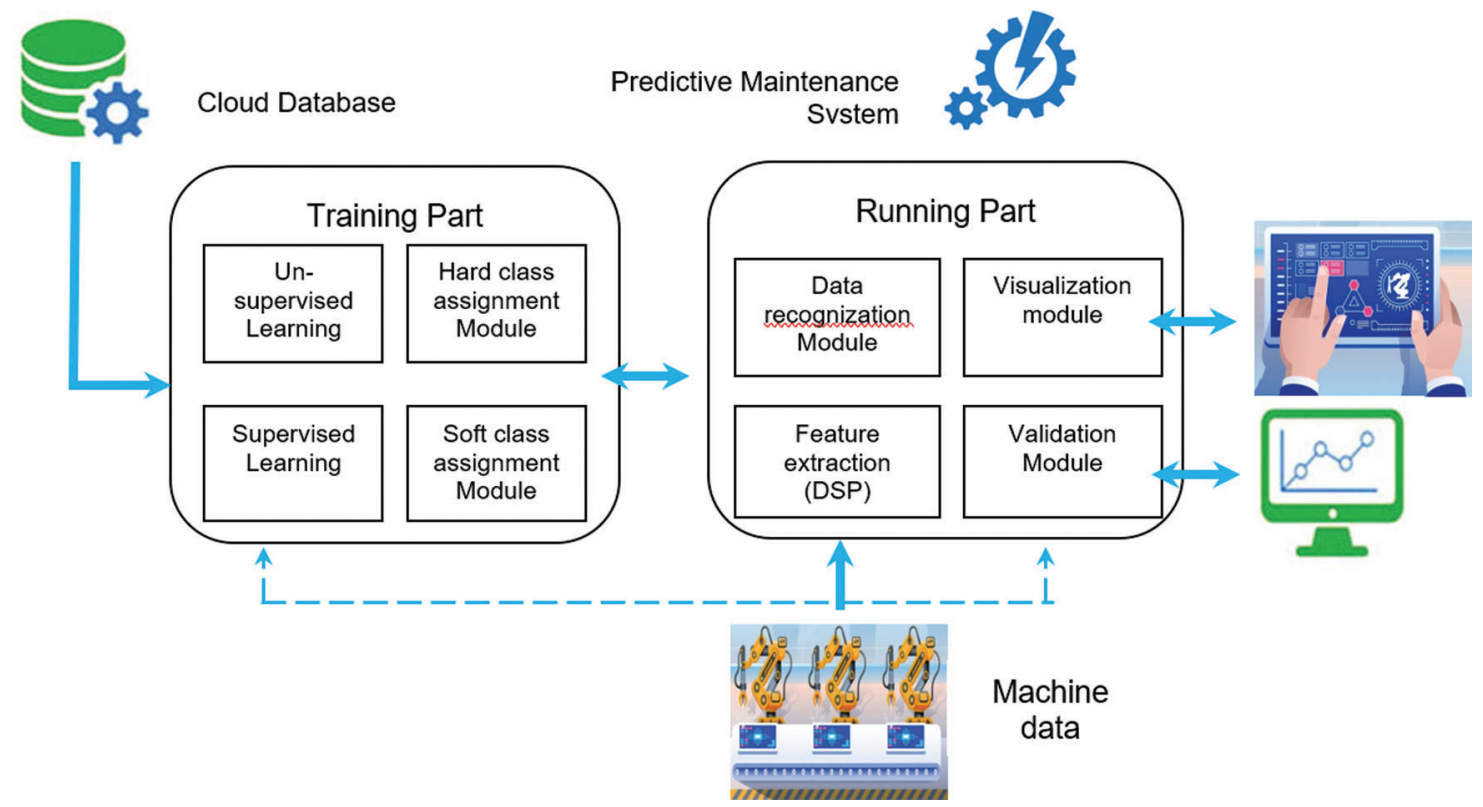

Figure 3: A hybrid machine learning strategy for predictive maintenance pilot

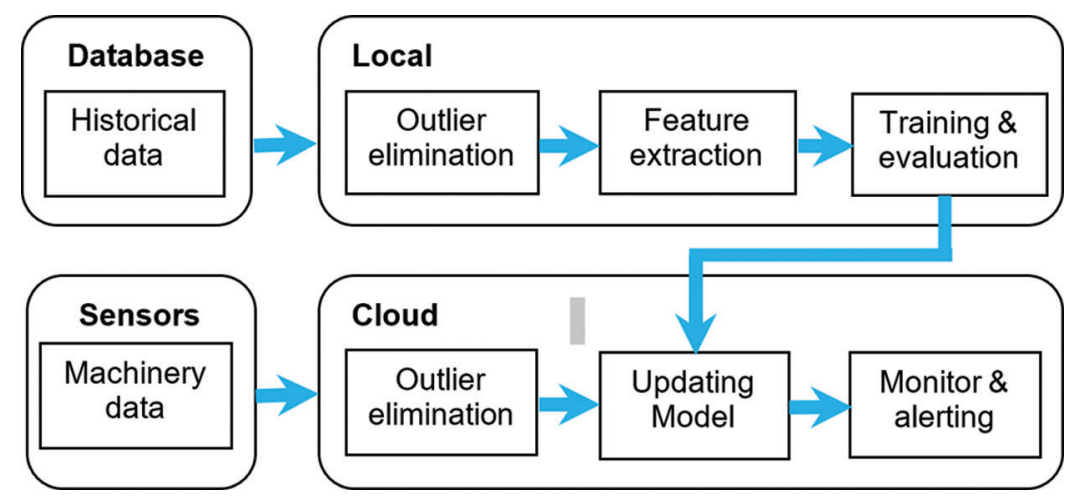

Figure 4: Data flow and processing

The sensor kit provides untrained dataset $\left(D_{U T}\right)$ directly extracted from the machine which is processed by unsupervised module where create a new dataset from feature extracted in the untrained data. Then, it is send to data stream analysis in which quality estimation of parameters carried. Based on the values, extract labelling is assigned to each dataset along with product Ids stored in the centre cloud. The class index is 
denoted by ' $k$ ' and $R_{k}=D_{N} / D_{T}$ where $\left(D_{T}\right)$ represents total number of instant dataset received and $\left(D_{N}\right)$ deals number of kth class of dataset. Then, evaluate the updated dataset after trained properly and it is denoted by $\left(X_{n}\right)$ where $X_{n} \varepsilon D_{U T}$ will be estimated for very instant data shared to the system. The resultant values gives appropriate adjustment needed in the processing parameters and bring into desired value; it will be continued for long period.

Evaluation (E) step:

$\gamma_{n k}=\frac{R_{k} S_{T}\left(X_{n} \mid \mu_{k} \sum_{k}\right)}{\sum_{j=1}^{K} R_{j} S_{T}\left(X_{n} \mid \mu_{j} \sum_{j}\right)}$

Adjustment (A) step:

$\mu_{k}^{\text {New }}=\frac{1}{S_{T_{k}}} \sum_{n=1}^{S_{T}} \gamma_{n k} X_{n}$

$\sum_{k}^{N e w}=\frac{1}{S_{T_{k}}} \sum_{n=1}^{S_{T}} \gamma_{n k}\left(X_{n}-\mu_{k}^{N e w}\right)\left(X_{n}-\mu_{k}^{N e w}\right)^{T}$

$R_{k}=D_{N} / D_{T}$

The maximum likelihood is estimated in the feature selection part for creating new dataset as given as follows:

$\ln p\left(X \mid \mu \sum R\right)=\sum_{n=1}^{S_{T}} \ln \left\{\sum_{k=1}^{K} R_{k} S_{T}\left(X_{n} \mid \mu_{k} \quad \sum_{k}\right)\right\}$

Initially, the predictive module has trained with predefined threshold value for parameter evaluation $P$ $\left[\mu_{k} \in D_{1 u}\right] \geq q$ and its start with soft-class assignation for unknown feature extraction using unsupervised learning module. It is clearly classified into required dataset for future references from Eq. (3). Then, it creates a new classifier input for earlier anomaly detection happens in the machine processing parameters. Later, it can easily accessible by maintenance system after verifying identification code generated randomly at the time of product investigation. Sequentially, running phase evaluates the instant product data with historical database to acquire a new set of data $P\left[X_{t} \varepsilon C_{k}\right]\left(X_{t} \varepsilon D_{2}\right)$ which helps for earlier detection of the product failure. The feature extraction module is involved to track the shapes and weight of the product and its value updating is stored after verifying condition that satisfies for adjusted condition $X_{n} \in D_{1 U}\left(D_{1}=D_{1 U} \cup D_{1 L}\right)$ in the both local and cloud space for future customer claims about the product failure in Eq. (5).

$\gamma_{n k}=\left\{\begin{array}{cc}\frac{R_{k} S_{T}\left(X_{n} \mid \mu_{k} \sum_{k}\right)}{\sum_{j=1}^{K} R_{j} S_{T}\left(X_{n} \mid \mu_{j} \sum_{j}\right)} & d_{n} \in D_{2 U} \\ 1(k=y(n)) & d_{n} \in D_{2 L}\end{array}\right.$

The digital display is mounted on each machine for their updated values and current status of number of machine analysed within processing time. It induces quality assurance of about manufacturing machine as well as product outlet. 


\section{Results and Discussion}

This section explores an evaluation of proposed predictive maintenance system in terms of the qualitative and quantitative form which is comprehensively compared with other machine learning approaches including unsupervised algorithms (K-Means \& Agglomerative clustering) and unsupervised algorithms (Random forest \& SVM) respectively. The experimental results include two major parts. Part A discusses the production efficiency of the manufacturing machine module at the operating time and ensures the quality outcome of the product before leave from the production line. The qualitative analysis is followed to evaluate the system performance of the proposed method. Part B presents the quantitative evaluation of the instant product data with historical database to acquire a new set of data which helps for earlier detection of the product failure. The feature extraction module is involved to track the shapes and weight of the product and its value is stored in the both local and cloud space for future customer claims about the product failure. The SimEvent toolbox of the MATLAB software is used for design the prototype model of the proposed system where qualitative and quantitative analysis is performed.

In general, many small scale manufacturing industries are not having product electronics diagnostics set up at each stage in the production line before launch into market. It is a complex task, very difficult to handle it make over into optimum solution data set, because, some product may take extra processing time to analysis its suspension adjustment for that if new equipment is used to calibrate operation, however, sometime it shows unable to interface with until advance cyclic time is initiated. Thereby, many industries follows separate supervisor is allotted to take care of quality checking but product failure is always there due to overhauling of the manual interruption. Since, they can do only static check not possible to do dynamic check. However, machine can do all type of product test including static and dynamic, once; the model is changed into automated. The simulation tool is involved to do all type of product testing and stored test data in the cloud for identifying new product failure pattern occurs. For that, separate environment is created to handle collected data, modify data for optimization which leads to give best set of data for the simulation unit. It accommodates multiple computing processes by running and training analysis on optimized data with parallel computing toolbox. Every product creates $\log$ file from each testing station. That means, let's take three testing wings where each one test 1000 products, then, $3000 \log$ file sets are logged. If you take log files of one product on one testing wing, it gives 200,000 lines of information. But, it contains only basic information like product details, test results and suspension time etc. To access all this data, a DOS-based batch-file is created which will be call whenever it required and distributes the details about each available product. The virtual vehicle model is developed and each product data is evaluated by interfacing testing DOS-based batch-file. It gives complete understanding of each product, how it works at different vehicle varying conditions. Based on that product database is created in the cloud. The statistical analysis on product evaluation in multiple dimension is carried by parallel computing toolbox which gives bar graphs of analysed data which includes testing times and vehicle variations. It is clearly mention in Fig. 5 Most of the simulation models consist of discrete-event (line data, queue and server) from predefined libraries. It is difficult to incorporate the vehicle actual functionality of the product and impact on the simulated system. In the proposed model, a m-script is created by accounting the dynamic parameters and then selects the baseline elements for SimEvents model. By this way, achieves an optimistic operation of the ML algorithm to adjust the model parameters and construct a new model for predicting earlier failures. It also enables interface models to identify root cause by the failure and corrective measurement is built in MATLAB. In Fig. 6 shows the SimEvents model generated by m-script with station and worker subsystems. In the generated model contains 1000 product, manual adjustment worker and product test on vehicle brought together at each station. The multiple single servers are involved in the individual product testing processes and estimate time spent at each product by an event-based random number feeder through log data for that station. 

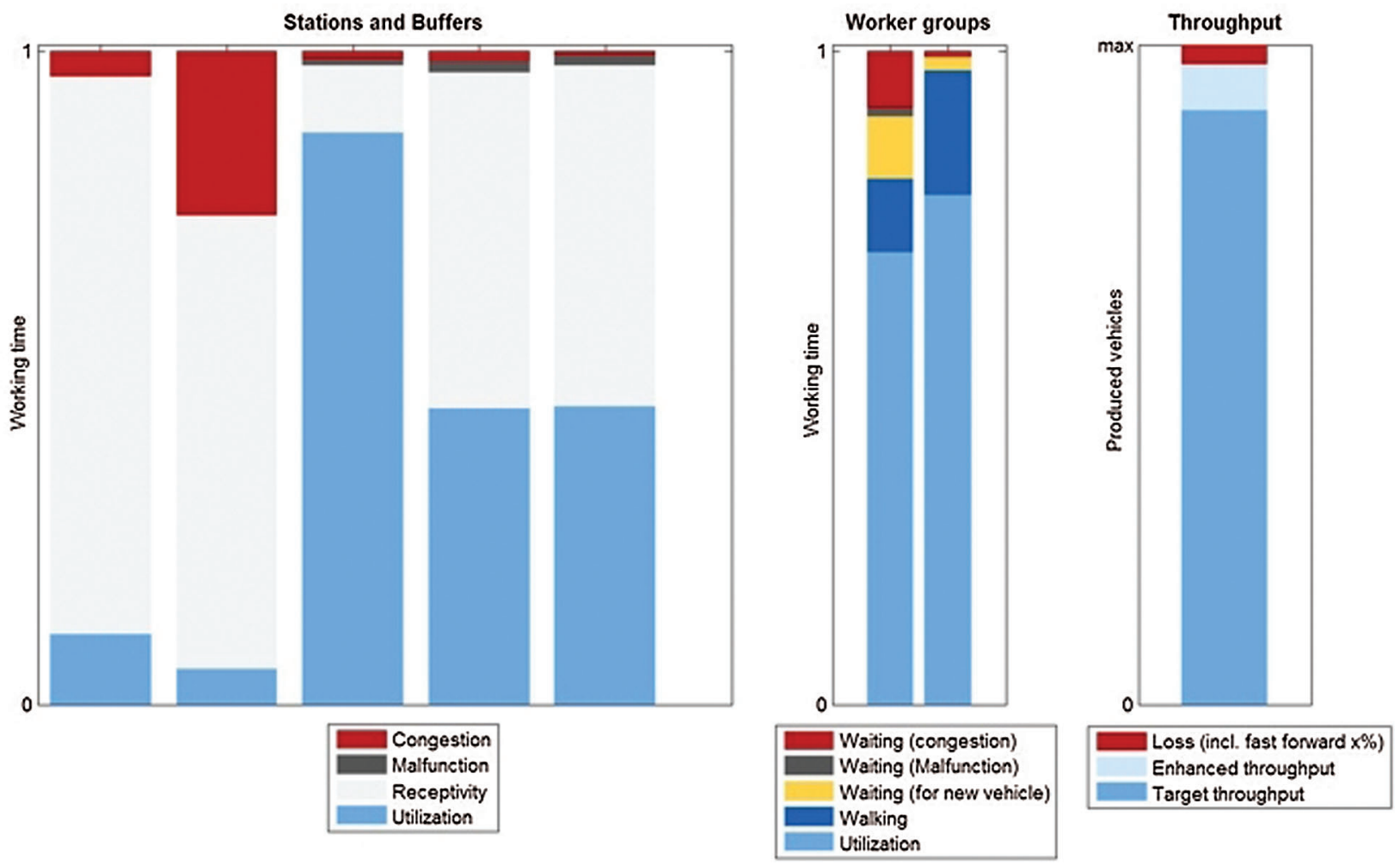

Figure 5: Product evaluation chart of the virtual vehicle model at different station wings

The hybrid machine learning strategy is carried out in the proposed IIoT framework for improving earlier detection of anomaly presence in the product as well as machine process deviation. In Fig. 3 gives a hybrid machine learning strategy for predictive maintenance pilot. The predictive maintenance system works under ML approach for segregating an optimistic dataset for control signal whenever finds. The logical functional behaviors and control strategy is generated using m-scripts incorporated into the model as S-Function blocks. The model stored the statistics data from each station, including how many products are processed, how long spent at each testing station, and how much time taken between successive number of stations. In Fig. 2 shows postprocess and visualize this data of the test product. In the proposed system replicate the interface and model generator existing in the industries. The simulations results of this model and compared its results with real-world results from the factory floor to validate the model and the model generation script. The proposed model is designed for two important scenarios (i) continuous tracking of product manufacturing machine in terms of process deviation and accuracy, (ii) extract the feature of the production line product with help of digital signal processing toolbox for effective analysis of product shape and weight. That is, first model involves analysis of machine performance using ML algorithms includes unsupervised learning (K-Means \& Agglomerative clustering) and supervised learning (Random forest \& SVM) respectively. It tracks the processing deviation by comparing the historical database from local as well as cloud dataset.

That is, if new data is identified for process deviation then it will be stored in the historical dataset in the cloud. In such case, un-supervised learning is trigged to get the data for future reference. Otherwise, if the datasets come from the local server, then, the supervised learning is availed. The main function of this phase is to identify the status of machines through ML based probabilistic model. The unsupervised learning is connected with soft-class decision module and supervised learning is connected with hardclass decision module. The initial class assignment process is carried by soft-class decision module of the 
unsupervised learning. It is very much careful about new occurs data. Similarly, consistency of classes analysis is performed by hard-class assignment of the supervised learning. In training phase, particularly focus to find process deviation of the manufacturing machine. It is obtained by evaluating three important parameters such as sensitivity, specificity and accuracy. The historical database is involved for comparing previous dataset with current dataset. if new data is identified for process deviation then it will be stored in the historical dataset in the cloud. In such case, un-supervised learning is trigged to get the data for future reference. Otherwise, if the datasets come from the local server, then, the supervised learning is availed. In Fig. 3 shows the performance analysis of proposed model at training part for different classes. The following manufacturing machine has considered for evaluation such as Laser Marking Robo Arm, Double Spindle Lathe and Horning machine.

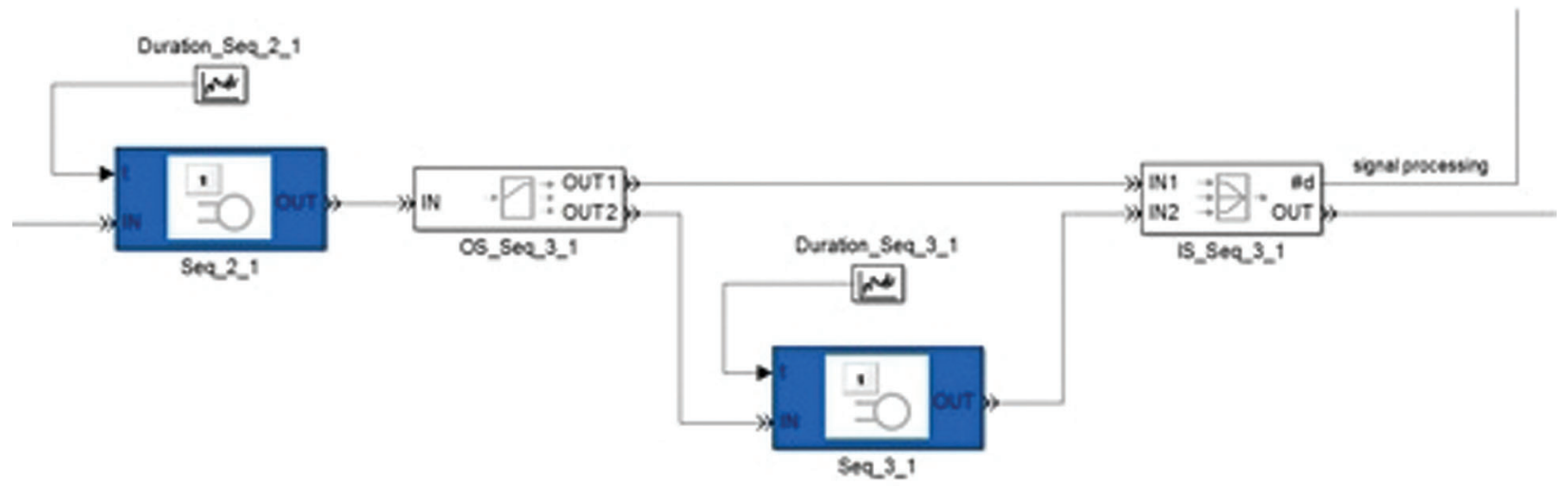

(a)

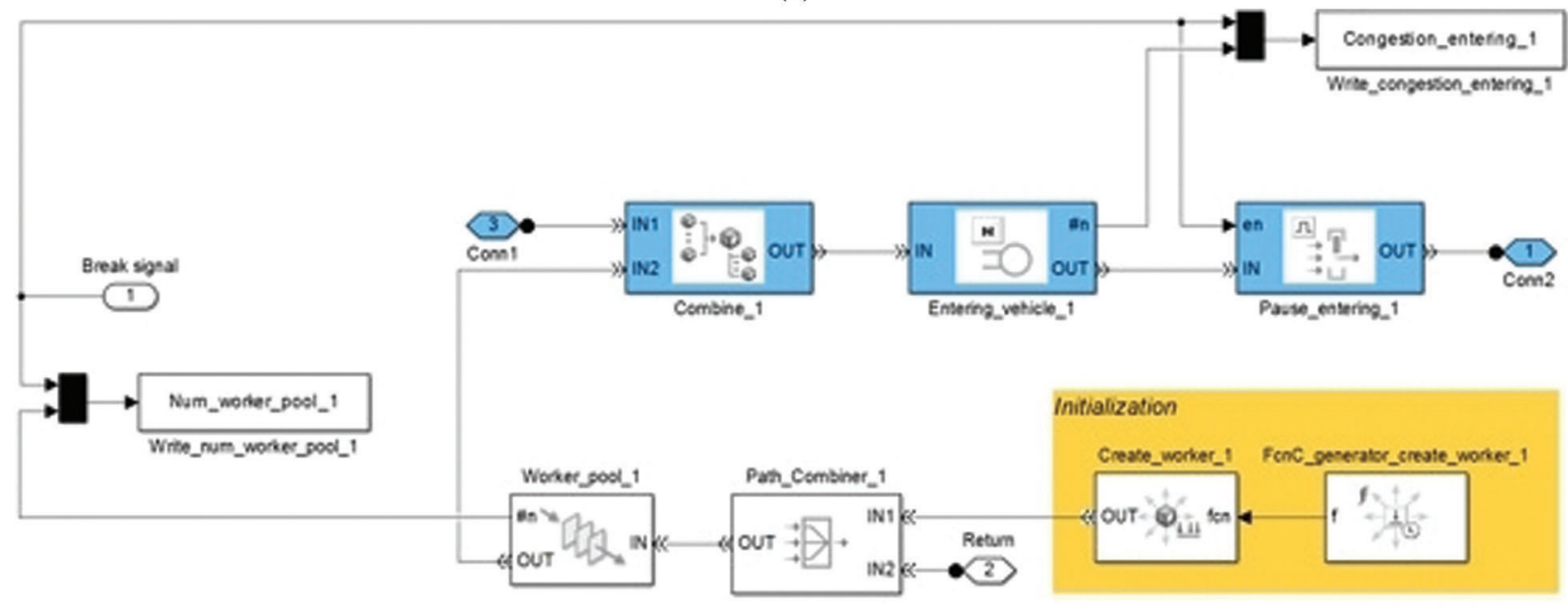

(b)

Figure 6: Strategical behaviours of the simevents modelled system (a) Training part, (b) Running part

The sensitivity values of above mentioned machine is reduced significantly due to proper classification of earlier fault detection occurs in the manufacturing model. Tabs. 2 and 3 conveys improved performance on specificity and accuracy, that means, in the existing model analysed minimum number of machines within given processing time. But, the proposed model enhanced its processing operation as compared with existing model in terms of number of manufacturing machine at the same processing time. It is inferred from Fig. 7 That is, the comparative analysis report is given as follows Laser Marking Robo Arm (10), 
Double Spindle Lathe (2) and Horning machine (3) which significantly improved with existing model Laser Marking Robo Arm (8), Double Spindle Lathe (1) and Horning machine (1). It is clearly mentioned in the Tab. 1 The main function of this phase is to identify the status of machines through ML based probabilistic model. In running phase, focus on acquiring a new set of data from instant product data which helps for earlier detection of the product failure. It is possible by using feature extraction module is involved to track the shapes and weight of the product and its value is stored in the both local and cloud space for future customer claims about the product failure. The supervised learning supports to track the earlier product deficiency because, maximum number of fault may occurs in the product shapes and weight. So, available dataset is more than sufficient to detect faulty part earlier. Hence, the supervised learning predominantly higher than unsupervised learning model which is mentioned in Tab. 4. It is clearly visible in the Fig. 8. Since, it is only useful when the new fault data is arrival after customers come for product compliant. Because, they may bring out new fault feature which can stored in the historical database for further reference.

Table 1: Comparative analysis of proposed hybrid ML model for maximization of machine lifetime

\begin{tabular}{lllll}
\hline Station wing no & Manufacturing machine & Existing no. & Proposed no. & Processing time (s) \\
\hline OP 15 & Laser Marking Robo Arm & 8 & 10 & 150 \\
OP 30 & Double Spindle Lathe & 1 & 2 & 36 \\
OP 45 & Horning machine & 1 & 3 & 45 \\
\hline
\end{tabular}

Table 2: Checking factors for process deviation of manufacturing machine at the training part

\begin{tabular}{llll}
\hline$\%$ of Max points & Sensitivity & Specificity & Accuracy \\
\hline 10 & 0.98 & 0.85 & 0.87 \\
20 & 0.97 & 0.87 & 0.91 \\
30 & 0.96 & 0.90 & 0.94 \\
40 & 0.96 & 0.94 & 0.95 \\
50 & 0.95 & 0.96 & 0.96 \\
60 & 0.95 & 0.96 & 0.97 \\
70 & 0.94 & 0.97 & 0.98 \\
80 & 0.91 & 0.98 & 0.99 \\
90 & 0.88 & 0.99 & 0.99 \\
\hline
\end{tabular}


Table 3: Checking factors for process deviation of manufacturing machine at the training part

\begin{tabular}{llll}
\hline \% of Max points & $R_{t r} / \operatorname{Max}$ & $R_{s v} / \operatorname{Max}$ & $R_{s v} / \operatorname{Max}$ \\
\hline 10 & 0.35 & 0.66 & 0.37 \\
20 & 0.41 & 0.72 & 0.39 \\
30 & 0.46 & 0.76 & 0.4 \\
40 & 0.50 & 0.80 & 0.44 \\
50 & 0.62 & 0.95 & 0.52 \\
60 & 0.73 & 0.86 & 0.58 \\
70 & 0.78 & 0.84 & 0.62 \\
80 & 0.82 & 0.82 & 0.66 \\
90 & 0.87 & 0.78 & 0.70 \\
\hline
\end{tabular}

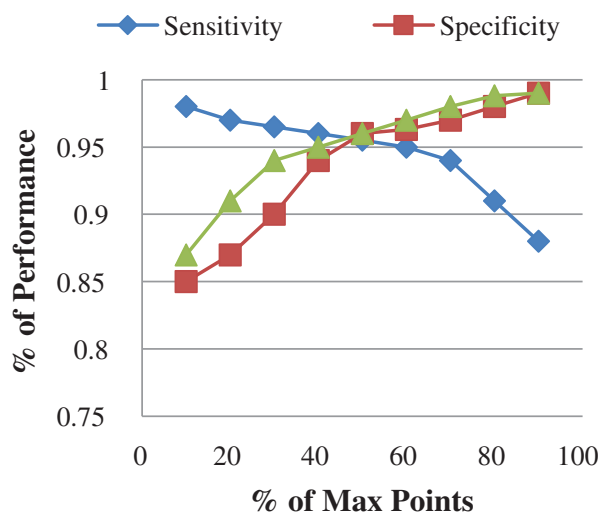

(a)

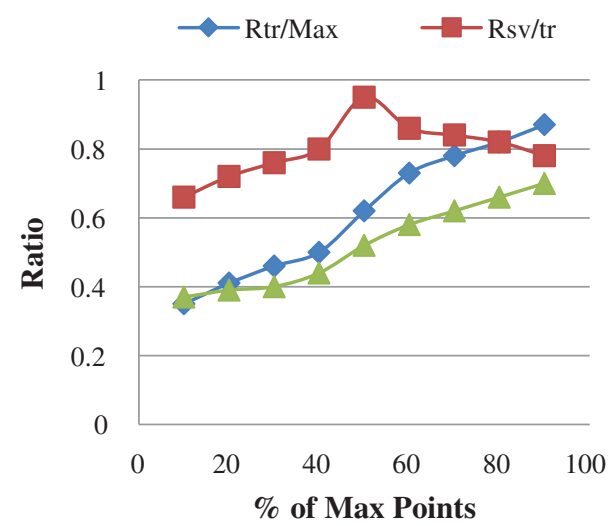

(b)

Figure 7: Performance of proposed model at training part for different classes (a) Process deviation (b) SVM ratio

Table 4: Comparative analysis of machine lifespan using unsupervised and supervised learning at the running part

\begin{tabular}{llllll}
\hline \multirow{2}{*}{$\%$ of Max points } & \multicolumn{2}{c}{ Unsupervised learning } & & \multicolumn{2}{c}{ Supervised learning } \\
\cline { 2 - 3 } & Existing scenario & Proposed scenario & & Existing scenario & Proposed scenario \\
\hline 10 & 0.994 & 0.989 & 0.998 & 0.992 \\
20 & 0.986 & 0.982 & 0.986 & 0.982 \\
30 & 0.972 & 0.966 & 0.977 & 0.973 \\
40 & 0.968 & 0.962 & 0.974 & 0.972 \\
50 & 0.957 & 0.96 & 0.967 & 0.964 \\
60 & 0.946 & 0.958 & 0.956 & 0.952 \\
70 & 0.945 & 0.913 & 0.952 & 0.949 \\
80 & 0.936 & 0.876 & 0.946 & 0.944 \\
90 & 0.914 & 0.852 & 0.934 & 0.929 \\
\hline
\end{tabular}




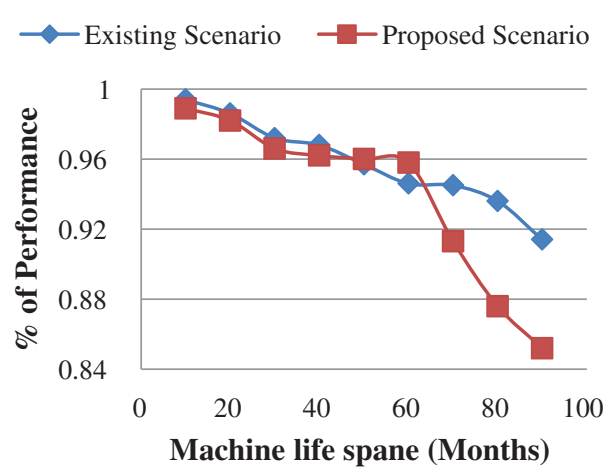

(a)

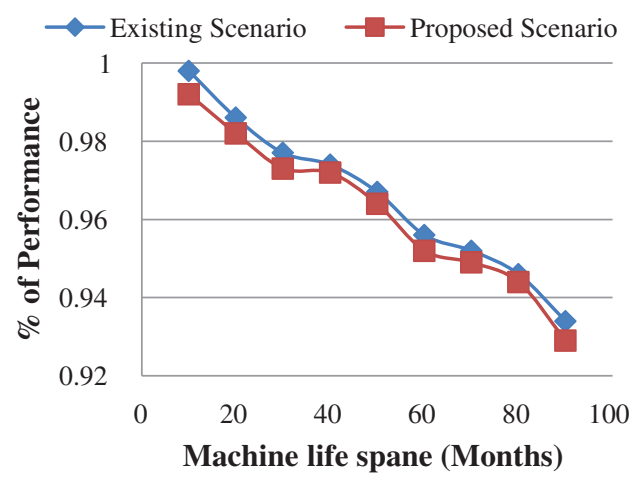

(b)

Figure 8: Performance of proposed model at running part for different classes (a) Unsupervised learning (b) Supervised learning

\section{Conclusion}

In this paper, an efficient predictive maintenance strategy is developed based on Machine Learning (ML) methods. It influenced hybrid ML model which combines unsupervised and supervised learning model. Hence, it has supported earlier prediction of failures and production line deviation which avoid shutdown in the production unit. There are several predictive maintenance units run behind in every production process at the backend which supported product substantial progression especially Accessories Manufacturers (AMs). It induced smart sensor kit for continuous collection of machine data instantly and ensured secured content saving at the centre cloud for further reference. The proposed model is designed for two important scenarios (i) training phase: continuous tracking of product manufacturing machine in terms of process deviation and accuracy, (ii) running phase: extract the feature of the production line product with help of digital signal processing toolbox for effective analysis of product shape and weight. In training phase, particularly focus to find process deviation of the manufacturing machine. It is obtained by evaluating three important parameters such as sensitivity, specificity and accuracy. In running phase, focus on acquiring a new set of data from instant product data which helps for earlier detection of the product failure. It is possible by using feature extraction module is involved to track the shapes and weight of the product and its value is stored in the both local and cloud space for future customer claims about the product failure. The supervised learning supports to track the earlier product deficiency because, maximum number of faults may occur in the product shapes and weight. So, available dataset is more than sufficient to detect faulty part earlier. Hence, the supervised learning predominantly higher than unsupervised learning model. Since, it is only useful when the new fault data is arrival after customers come for product compliant. Because, they may bring out new fault feature which can stored in the historical database for further reference. The proposed predictive methodology has been tested in terms of earlier anomaly detection, production line accuracy \& machinery efficiency and compared with other existing ML based predictive maintenance approaches.

Funding Statement: The authors received no specific funding for this study.

Conflicts of Interest: The authors declare that they have no conflicts of interest to report regarding the present study. 


\section{References}

[1] F. Shrouf, J. Ordieres and G. Miragliotta, "Smart factories in industry 4.0: A review of the concept and of energy management approached in production based on the Internet of Things paradigm," in 2014 IEEE Int. Conf. on Industrial Engineering and Engineering Management, pp. 697-701, 2014.

[2] P. Suresh, K. Aanandhasaravanan, C. Iwendi, E. Ibeke and G. Srivastava, "An artificial intelligence-based quorum system for the improvement of the lifespan of sensor networks," IEEE Sensors Journal, vol. 21, no. 12, pp. 1-13, 2021.

[3] L. Da Xu, W. He and S. Li, "Internet of things in industries: A survey," IEEE Transactions on Industrial Informatics, vol. 10, no. 4, pp. 2233-2243, 2014.

[4] K. Zhou, T. Liu and L. Zhou, "Industry 4.0: Towards future industrial opportunities and challenges," 2015 12th Int. Conf. on Fuzzy Systems and Knowledge Discovery (FSKD), pp. 2147-2152, 2015.

[5] J. Guth, U. Breitenbücher, M. Falkenthal, P. Fremantle, O. Kopp et al., "A detailed analysis of IoT platform architectures: Concepts, similarities, and differences," In Internet of Everything: Springer, pp. 81-101, 2018.

[6] C. Federico, B. Stefano, S. Claudio, R. Enrico, M. Luca et al., "Industrial internet of things monitoring solution for advanced predictive maintenance applications," Journal of Industrial Information Integration, vol. 7, pp. 4-12, 2017.

[7] E. Sisinni, A. Saifullah, S. Han, U. Jennehag and M. Gidlund, "Industrial internet of things: Challenges, opportunities, and directions," IEEE Transactions on Industrial Informatics, vol. 14, no. 11, pp. 4724-4734, 2018.

[8] Y. He, C. Gu, Z. Chen and X. Han, "Integrated predictive maintenance strategy for manufacturing systems by combining quality control and mission reliability analysis," International Journal of Production Research, vol. 55, no. 19, pp. 5841-5862, 2017.

[9] M. Revathi, P. Suresh, C. Chinmay and U. Saravanakumar, "Improved performance on seizure detection in an automated electroencephalogram signal under evolution by extracting entropy feature," Multimedia Tools and Applications, vol. 80,. no.17, pp. 1-18, 2021.

[10] D. P. Filev, R. B. Chinnam, F. Tseng and P. Baruah, "An industrial strength novelty detection framework for autonomous equipment monitoring and diagnostics," IEEE Transactions on Industrial Informatics, vol. 6, no. 4, pp. 767-779, 2010.

[11] Y. Lu, "Industry 4.0: A survey on technologies, applications and open research issues," Journal of Industrial Information Integration, vol. 6, no. 1, pp. 1-10, 2017.

[12] S. Landset, T. M. Khoshgoftaar, A. N. Richter and T. Hasanin, "A survey of open-source tools for machine learning with big data in the hadoop ecosystem," Journal of Big Data, vol. 2, no. 1, pp. 24, 2015.

[13] Y. Lei, F. Jia, J. Lin, S. Xing and S. X. Ding, "An intelligent fault diagnosis method using unsupervised feature learning towards mechanical big data," IEEE Transactions on Industrial Electronics, vol. 63, no. 5, pp. 31373147, 2016.

[14] E. Uhlmann, A. Laghmouchi, C. Geisert and E. Hohwieler, "Decentralized data analytics for maintenance in industries 4.0," Procedia Manufacturing, vol. 11, pp. 1120-1126, 2017.

[15] J. Wang, L. Zhang, L. Duan and R. X. Gao, "A new paradigm of cloud-based predictive maintenance for intelligent manufacturing," Journal of Intelligent Manufacturing, vol. 28, no. 5, pp. 1125-1137, 2017.

[16] P. Suresh, U. Saravanakumar, C. Iwendi, S. Mohan and G. Srivastav, "Field-programmable gate arrays with low power vision system using dynamic switching," Computers \& Electrical Engineering, vol. 90, no. 1, pp. 1-13, 2021.

[17] C. H. Glock and E. H. Grosse, "Decision support models for production ramp-up: A systematic literature review," International Journal of Production Research, vol. 53, no. 21, pp. 6637-6651, 2014.

[18] S. M. Erfani, S. Rajasegarar, S. Karunasekera and C. Leckie, "High-dimensional and large-scale anomaly detection using a linear one-class SVM with deep learning," Pattern Recognition, vol. 58, pp. 121-134, 2016.

[19] V. Solanki, P. K. Dhall and S. Rohit, "An IoT based predictive connected car maintenance." International Journal of Interactive Multimedia \& Artificial Intelligence, vol. 4,. no. 3, pp. 16-22, 2017. 
IASC, 2022, vol.31, no.3

[20] A. Chowdhury, T. Banerjee, T. Chakravarty and P. Balamuralidhar, "Smartphone based sensing enables automated vehicle prognosis," 2015 9th International Conference on Sensing Technology (ICST), pp. 452455, 2015.

[21] Y. Li, E. Pan and Z. Chen, "Considering machine health condition in jointly optimizing predictive maintenance policy and X-bar control chart." 2017 Int. Conf. on Grey Systems and Intelligent Services (GSIS), pp. 328-337, 2017.

[22] N. Herr, J. Nicod, C. Varnier, N. Zerhouni and P. Dersin, "Predictive maintenance of moving systems." 2017 Prognostics and System Health Management Conference (PHM-Harbin), pp. 1-6, 2017.

[23] K. A. Kaiser and N. G. Gebraeel, "Predictive maintenance management using sensor-based degradation models." IEEE Transactions on Systems, Man, and Cybernetics-Part a: Systems and Humans, vol. 39, no. 4, pp. 840-849, 2009.

[24] Satta R. et al. "A dissimilarity-based approach to predictive maintenance with application to HVAC systems." ArXiv abs/1701.03633, 2017.

[25] P. Ramu, S. P. Vimal, P. Suresh, U. Saravanakumar, V. Sethuraman, "Electrochemically deposited porous graphene-polypyrrole-polyphenol oxidase for dopamine biosensor," Electroanalysis (Willey), vol. 33, no. 3, pp. 1-12, 2021. 